\title{
Radiative Processes in the Stable Boundary Layer: Part I. Radiative Aspects
}

\section{J. M. Edwards}

Published online: 27 June 2009

(C) Springer Science+Business Media B.V. 2009

\section{Erratum to: Boundary-Layer Meteorol (2009) 131:105-126 DOI 10.1007/s10546-009-9364-8}

I am grateful to Prof A. S. Vasudeva Murthy for drawing my attention to errors in Eqs. 2, 3 and 7 in Sect. 2.1 of this article. These originated from an earlier version of the work and were subsequently not detected. I would like to apologise for any confusion or inconvenience that has been caused. However, it should be noted that the following argument about the role of the surface emissivity is unaffected by the corrections and that the subsequent results and conclusions presented are also unchanged.

Equation 2 should read:

$$
F_{v}^{-}(z)=\pi B_{v}(z)-\mathscr{T}_{v}(z, \infty) \pi B_{v}(\infty)+\int_{z}^{\infty} \mathscr{T}_{v}\left(z, z^{\prime}\right) \frac{d}{d z^{\prime}} \pi B_{v}\left(z^{\prime}\right) d z^{\prime},
$$

Equation 3 should read:

$$
\begin{aligned}
F_{v}^{+}(z)= & F_{v}^{+}(0) \mathscr{T}_{v}(0, z)+\pi B_{v}(z)-\mathscr{T}_{v}(0, z) \pi B_{v}(0) \\
& -\int_{0}^{z} \mathscr{T}_{v}\left(z^{\prime}, z\right) \frac{d}{d z^{\prime}} \pi B_{v}\left(z^{\prime}\right) d z^{\prime}
\end{aligned}
$$

and these forms should be carried forward into Eq. 7, which should read:

The online version of the original article can be found under doi:10.1007/s10546-009-9364-8.

J. M. Edwards $(\bowtie)$

Met Office, FitzRoy Road, Exeter EX1 3PB, UK

e-mail: john.m.edwards@metoffice.gov.uk 


$$
\begin{aligned}
F_{v}^{+}(z)= & \varepsilon_{F v} \pi B_{v}(0) \mathscr{T}_{v}(0, z)+\left(1-\varepsilon_{F v}\right) \mathscr{T}_{v}(0, z) \\
& \left\{\pi B_{v}(0)-\mathscr{T}_{v}(0, \infty) \pi B_{v}(\infty)+\int_{0}^{\infty} \mathscr{T}_{v}\left(0, z^{\prime}\right) \frac{d}{d z^{\prime}} \pi B_{v}\left(z^{\prime}\right) d z^{\prime}\right\} \\
& +\pi B_{v}(z)-\pi B_{v}(0) \mathscr{T}_{v}(0, z)-\int_{0}^{z} \mathscr{T}_{v}\left(z^{\prime}, z\right) \frac{d}{d z^{\prime}} \pi B_{v}\left(z^{\prime}\right) d z^{\prime}
\end{aligned}
$$

